\title{
Les lipides, protéines et glucides stimulent la sécrétion de cholécystokinine intestinale chez le porc
}

\author{
JC Cuber ${ }^{1,2 \star}$, C Bernard $^{2}$, F Levenez ${ }^{1}$, JA Chayvialle ${ }^{2}$ \\ avec la collaboration technique de T Gibard, G Brachet, F Cointepas \\ 1 INRA, Station de physiologie de la nutrition, Centre de recherches de Jouy-en-Josas, \\ 78350 Jouy-en-Josas; \\ 2 INSERM U 45, hôpital Edouard -Herriot, pavillon H bis, 69437 Lyon Cedex 3
}

(Reçu le 12 octobre 1989; accepté le 30 janvier 1990)

\begin{abstract}
Résumé - La prise alimentaire stimule la libération de cholécystokinine (CCK) chez le porc, mais la contribution de chaque classe de nutriments à la réponse de CCK n'a pas encore été établie dans cette espèce. Six porcs mâles castrés, d'un poids moyen de $50 \mathrm{~kg}$, ont été munis d'une fistule duodénale pour perfuser les nutriments, de cathéters dans la veine porte (VP) et dans une artère carotide $(A C)$ pour prélever les échantillons sanguins. Après un jeûne de 24 heures, $1000 \mathrm{ml}$ de solutions isotoniques contenant $440 \mathrm{kcal}$ de glucides (hydrolysat d'amidon) ou de protéines (hydrolysat de caséine) ou de lipides (Intralipide) étaient perfusés pendant $60 \mathrm{~min}$ après une période basale de $60 \mathrm{~min}$ pendant laquelle du sérum physiologique était perfusé. Des échantillons de sang porte et périphérique étaient prélevés à intervalles de $15 \mathrm{~min}$ en vue du dosage radioimmunologique de CCK. Les graisses ont provoqué une augmentation importante de l'immunoréactivité CCK dans la veine porte (pic à $76,6 \pm 12,2 \mathrm{pM}$ avec un taux basal de 10,8 $\pm 1,2 \mathrm{pM}$ ) et dans le sang périphérique (pic à $46,7 \pm 8,4 \mathrm{pM}$ avec une valeur basale de $9,1 \pm 1,0 \mathrm{pM}$ ). L'hydrolysat protéique n'a induit qu'une augmentation transitoire du taux de CCK plasmatique durant la première demi-heure (VP : pic à 40,1 \pm $5,0 \mathrm{pM}$ avec un taux basal de 11,9 $\pm 1,4 \mathrm{pM} ; \mathrm{AC}: 31,8 \pm 4,0 \mathrm{pM}$ avec un basal de $8,5 \pm 0,8 \mathrm{pM})$. Le caractère transitoire de la réponse de CCK aux protéines pourrait représenter la conséquence de la libération de somatostatine intestinale. L'hydrolysat d'amidon a eu un effet brusque sur la concentration de CCK plasmatique qui, rapidement, a atteint une valeur plateau dans la veine porte $(52,5 \pm$ $13,1 \mathrm{pM}$ à partir d'un basal de 11,9 $\pm 1,4 \mathrm{pM})$ et dans le sang artériel $(35,4 \pm 8,0 \mathrm{pM}$ avec une valeur basale de $8,5 \pm 0,8 \mathrm{pM})$. L'administration dans la lumière intestinale de glucose $(280 \mathrm{mM})$ a également produit une sécrétion soutenue de CCK. Il est conclu que, chez le porc, les lipides, les glucides et à un moindre degré les protéines stimulent la sécrétion de CCK intestinale.
\end{abstract}

cholécystokinine / porc / lipide / protéine / glucide

Summary - Intraduodenal infusion of fats, proteins and carbohydrates stimulates the release of intestinal cholecystokinin in the pig. Food intake enhances the release of intestinal cholecystokinin (CCK) in the pig but the contribution of individual nutrients to the CCK response has not yet been established in this species. Six hogs (mean weight $50 \mathrm{~kg}$ ) were fitted with a duodenal fistu-

\footnotetext{
* Correspondance et tirés à part
} 
la for instillation of nutrients and with portal (PV) and carotid (CA) catheters for blood sampling. After a 24- $h$ fast, the animals received $1000 \mathrm{ml}$ of isotonic solution containing $440 \mathrm{kcal}$ of carbohydrate (starch hydrolysate), or of protein (casein hydrolysate) or fat (Intralipid) or a control saline solution by 60-min intraduodenal perfusion after a 60-min control period during which the animals received saline. Portal and peripheral blood samples were collected at 15-min intervals for CCK radioimmunoassay. Intraduodenal perfusion of fat provoked a sharp increase in CCK-Like immunoreactivity (CCK-LI) in PV (peak $76.6 \pm 12.2 \mathrm{pM}$ from basal $10.8 \pm 1.2 \mathrm{pM}$ ) and in peripheral blood (peak $46.7 \pm 8.4 \mathrm{pM}$ from basal $9.1 \pm 1.0 \mathrm{pM}$ ). The protein hydrolysate induced a transient increase in plasma CCK-LI during the first 30 min of intestinal perfusion ( $P V$ : peak $40.1 \pm 5.0 \mathrm{pM}$ from basal 11.9 $\pm 1.4 \mathrm{pM}$; CA: $31.8 \pm 4.0 \mathrm{pM}$ from basal $8.5 \pm 0.8 \mathrm{pM}$ ). The transient effect of proteins on CCK release might reflect the consequence of somatostatin release from intestinal stores. Starch hydrolysate promptly raised plasma CCK-LI level to a plateau value ( $P V: 52.5 \pm 13.1 \mathrm{pM}$ from basal 11.9 $\pm 1.4 \mathrm{pM} ; C A: 35.4 \pm 8.0$ from basal $8.5 \pm 0.8 \mathrm{pM})$. Luminal administration of glucose $(280 \mathrm{mM})$ also elicited a well-sustained CCK release. It is concluded that in the pig, fats, carbohydrates and, to a lesser extent, proteins stimulate the release of intestinal CCK.

cholecystokinin / pig / fat / protein / carbohydrate

\section{INTRODUCTION}

La cholécystokinine (CCK) est un peptide qui a été purifié initialement à partir de la muqueuse duodénale porcine sous une forme contenant 33 acides aminés (Mutt et Jorpes, 1968). Par la suite, d'autres formes de CCK ont été identifiées dans la muqueuse intestinale et le cerveau (CCK83, CCK58, CCK22, CCK8) chez le porc mais également chez l'homme, le chien et le rat (Eng et al, 1984; Reeve et al, 1984; Tatemoto et al, 1984; Eberlein et al, 1989). Ces peptides ont comme caractéristique une partie C-terminale commune comprenant une tyrosine sulfatée en position 7 (à partir de l'extrémité C-terminale) et une phénylalanine amidée C-terminale. Toute l'activité biologique de la CCK est retenue dans l'heptapeptide $\mathrm{C}$-terminal (Ondetti et al, 1970). A l'image de la CCK intestinale, la CCK circulante est également hétérogène chez le porc (Cantor et Rehfeld, 1989; Cuber et al, 1989b) et d'autres espèces (Eysselein et al, 1984; Liddle et al, 1985; Fölsch et al, 1987). L'immunoréactivité CCK augmente dans le plasma après l'ingestion d'un repas chez l'homme (Liddle et al, 1985), chez le chien
(Chang et Chey, 1983), chez le rat (Liddle et al, 1984) et chez le porc (Cuber et al, 1989b) avec des conséquences variables selon l'espèce sur diverses fonctions digestives : inhibition de l'évacuation gastrique, stimulation de la sécrétion de pepsinogène, stimulation de la motricité intestinale et des contractions de la vésicule biliaire, ainsi qu'une stimulation du pancréas endocrine et exocrine (Walsh, 1987). D'une manière générale, les différentes formes moléculaires de la CCK biologiquement active exercent, sur une base molaire, des effets comparables sur les tissus cibles (Walsh, 1987). La CCK est donc l'une des hormones intestinales les plus importantes du tube digestif.

Cependant, les mécanismes de contrôle de la libération de ce peptide sont assez mal connus bien qu'un faisceau d'informations commence à émerger au moins chez le rat (Louie et al, 1986; Cuber et al, 1989d). La cellule endocrine I est, par son pôle basal, au contact d'un riche réseau neuropeptidergique, tandis qu'au pôle apical, elle reçoit des informations provenant du chyme intestinal dont les nutriments constituent une part importante. Les lipides, les protéines ainsi que leurs pro- 
duits d'hydrolyse sont de puissants stimulants de la sécrétion de CCK chez l'homme (Liddle et al, 1985; Miazza et al, 1985), tandis que seuls la caséine et l'inhibiteur trypsique du soja (Liddle et al, 1986) ou les hydrolysats protéiques (Cuber et al, 1989 e) induisent une sécrétion de CCK chez le rat. Ces résultats suggèrent d'importantes variations interespèces eu égard à la capacité des différents nutriments à stimuler la sécrétion de CCK. Le présent travail a été entrepris afin d'identifier les nutriments susceptibles de stimuler la sécrétion de CCK chez le porc en croissance.

\section{MATÉRIEL ET MÉTHODES}

\section{Préparation chirurgicale}

Sous anesthésie générale au fluothane, 6 porcs mâles castrés de race Large White d'un poids vif moyen de $55 \pm 3 \mathrm{~kg}$ et provenant de l'élevage du département de nutrition, INRA (La Minière) ont été équipés d'une fistule duodénale permanente (tube silastic ${ }^{\circledR}$ de diamètre intérieur 2,64 $\mathrm{mm}$ et de diamètre extérieur $4,88 \mathrm{~mm}$ ) placée $15 \mathrm{~cm}$ en aval du pylore. Un cathéter en polyéthylène (di $1,77 \mathrm{~mm}$, de $2,88 \mathrm{~mm}$ ) et un autre en silastic (di $1,86 \mathrm{~mm}$, de $3,15 \mathrm{~mm}$ ) ont été placés dans la veine porte et dans une artère carotide, respectivement. Les cathéters sanguins étaient entretenus journellement par rinçage avec du sérum physiologique contenant de l'héparine. La période expérimentale débutait après une période de récupération postopératoire d'environ 10 jours.

\section{Protocole expérimental}

Après une période de jeûne de $24 \mathrm{~h}$ pendant laquelle les animaux n'ont reçu que de l'eau à volonté, divers nutriments ont été perfusés par voie duodénale en quantités isocaloriques ( 440 kcal) à un débit de $1000 \mathrm{ml} / \mathrm{h}$, pendant $1 \mathrm{~h}$ après une période basale de même durée lors de laquelle seul du sérum physiologique était introduit dans la lumière intestinale. Une seule substance était perfusée par test, la nature de chacune d'elles étant choisie par tirage au sort. Chaque journée expérimentale était suivie d'une période de récupération de 2 jours. $\mathrm{Le} \mathrm{pH}$ et l'osmolarité des solutions test étaient ajustés à 7,0 et $300 \mathrm{mOsm} / \mathrm{l}$, respectivement, immédiatement avant leur introduction dans la lumière intestinale. La solution de glucides consistait en (i) un hydrolysat d'amidon (Caloreen, Laboratoires Sopharga, Puteaux) contenant des polymères ne renfermant pas plus de 7 unités glucose ou (ii) une solution de glucose à $5 \%$ (Laboratoire Aguettant, Lyon). La source protéique était un hydrolysat de protéines du lait (Laboratoires Sopharga) contenant $50 \%$ de peptides de poids moléculaire inférieur à $600,30 \%$ de peptides de poids moléculaire compris entre 600 et 1500 . Les $20 \%$ de peptides résiduels avaient un poids moléculaire supérieur à 1500 mais inférieur à 6000 (Rérat et al, 1988). Les lipides étaient composés d'huile de soja et de lécithine d'œuf (Intralipide ${ }^{3}$, Laboratoires Kabivitrum, Noisy-leGrand). Cinq $\mathrm{ml}$ de sang portal et périphérique collectés pendant les périodes basale et de stimulation étaient recueillis toutes les $15 \mathrm{~min}$ dans des tubes maintenus dans la glace et contenant $10 \mathrm{U}$ d'héparine et $500 \mathrm{kU}$ de Trasy$\mathrm{lol} \otimes / \mathrm{ml}$ de sang. Après centrifugation à $2000 \mathrm{~g}$ pendant $15 \mathrm{~min}$, le plasma était prélevé et maintenu à $-20^{\circ} \mathrm{C}$ en vue du dosage radioimmunologique de la CCK.

\section{Dosage radioimmunologique de la cholécystokinine}

La description complète de la méthode de dosage de la CCK plasmatique a été rapportée précédemment (Miazza et al, 1985). Brièvement, l'antisérum $67 \mathrm{H}$ a été obtenu chez un lapin immunisé avec la CCK33 (don du $\operatorname{Pr} V$ Mutt, Karolinska Institute, Stockholm) couplé à l'albumine avec du 1-éthyl-3-(3-diméthyl-aminopropyl)-carbodiimide (Sigma chemical Ltd, Saint Louis, Etats-Unis). Cet antisérum reconnaît à équivalence CCK 33, CCK39 et les formes moléculaires carboxy-terminales sulfatées de 9 amino acides ou plus. La réactivité de l'octapeptide C-terminal sulfaté était de $30 \%$, celle du peptide désulfaté et celle de la gastrine étant inférieures à $3 \%$. Le traceur, préparé par cou- 
plage du décapeptide C-terminal sulfaté (don du Pr Wünsch) au réactif de Bolton et Hunter monoiodé (Nex 120, New England Nuclear, Dupont de Nemours, Paris), a été purifié par chromatographie liquide à haute performance en phase inverse (Bondapak, C18) selon Fourmy et al (1982). Les plasmas ont été testés contre une courbe standard préparée avec du plasma provenant de chaque animal et épuré au charbon Norit A. La récupération de la CCK33 et celle de la CCK8 ont varié entre 62 et $80 \%$. La variation intradosage était de $9 \%$, la variation interdosages de $13,5 \%$.

\section{Expression des résultats}

Toutes les valeurs des taux plasmatiques de CCK sont exprimées en pM accompagnées de leurs écarts types réduits. Toutes les données ont été exploitées en utilisant le test $t$ de Student pour mesures appariées.

\section{RÉSULTATS}

A l'état basal, la concentration plasmatique de CCK était faible et stable avec des valeurs à $8 \mathrm{pM}$ dans le sang périphérique et à $10 \mathrm{pM}$ dans le sang portal. L'administration de sérum physiologique dans le duodénum n'a pas modifié les taux basaux de CCK. En revanche, l'instillation de lipides a eu pour conséquence une augmentation progressive de la concentration de CCK dans le sang porte atteignant un maximum de 76,6 $\pm 12,2 \mathrm{pM}(P<0,05)$ à l'arrêt de la perfusion, une décroissance régulière $\mathrm{du}$ taux de CCK étant observée par la suite sans qu'un retour aux valeurs basales ne soit observé à la fin de la période expérimentale (taux de CCK à 26,5 \pm 4,6 pM, $P<0,05)$. Un profil similaire mais moins marqué est observé pour la concentration de CCK périphérique (pic à 46,7 $\pm 8,4 \mathrm{pM}$ à l'arrêt de la stimulation et retour à une valeur de $21,8 \pm 2,8 \mathrm{pM}$ à la fin du test) (fig 1).
Une augmentation transitoire des taux de CCK portale et périphérique est observée au cours des 15 premières minutes de perfusion de l'hydrolysat protéique avec un pic de CCK à 40,1 $\pm 5,0$ pM possédant une valeur basale de 11,9 $\pm 1,4 \mathrm{pM}$ dans le sang portal $(P<0,05)$ et un pic de CCK à $31,8 \pm 4,0 \mathrm{pM}$ avec un taux basal à $8,5 \pm$ $0,8 \mathrm{pM}$ dans le sang artériel $(P<0,05)$. Un retour rapide à des valeurs proches du taux basal est observé par la suite en clépit du fait que la perfusion de l'hydrolysat protéique est poursuivie (fig 2).

En revanche, l'administration luminale d'un hydrolysat d'amidon a provoqué une augmentation immédiate du taux de CCK portale (pic significatif à $65,1 \pm 15,5 \mathrm{pM}$ avec un basal à 11,9 $\pm 1,4 \mathrm{pM}$ ) reflétée dans le sang périphérique avec un pic à $36,9 \pm 10,2 \mathrm{pM}$ à partir d'un taux basal à $8,5 \pm 0,8 \mathrm{pM}(P<0,05)$. Les taux de CCK portale et périphérique se sont maintenus à des niveaux stationnaires pendant toute la période de stimulation avec des valeurs moyennes de $45 \mathrm{pM}$ et $35 \mathrm{pM}$, respectivement. L'interruption de la perfusion de l'hy-

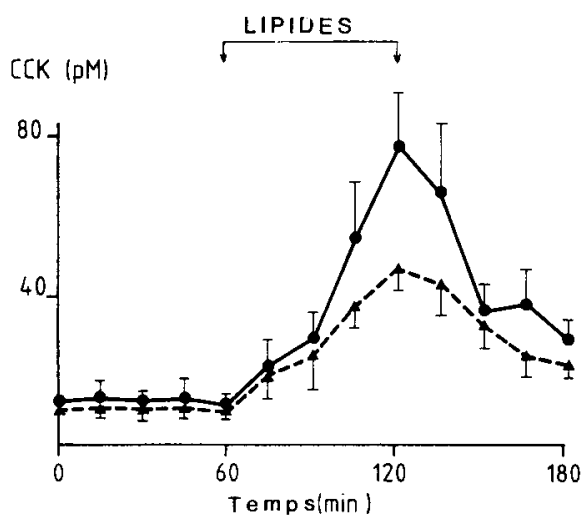

Fig 1. Effet de l'instillation duodénale de lipides (intralipide) sur les concentrations plasmatiques de CCK (pM) dans la veine porte $(-\bullet)$ et dans le sang artériel ( $\mathbf{A}-\mathbf{A})$. Chaque point représente la moyenne \pm écart-type réduit des valeurs obtenues chez 6 porcs. 


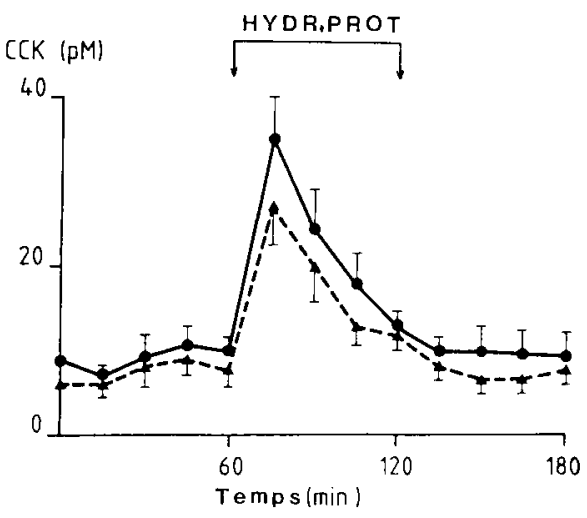

Fig 2. Evolutions en fonction du temps des concentrations plasmatiques de CCK chez 6 porcs ayant reçu une perfusion luminale d'un hydrolysat protéique. Les sigles sont identiques à ceux de la figure 1 .

drolysat d'amidon a eu pour conséquence un retour progressif des taux plasmatiques de CCK à des niveaux proches des concentrations basales dans l'intervalle d'1 heure (fig 3). La perfusion luminale d'une solution de glucose à $5 \%$ a provoqué une élévation immédiate de la concentration de CCK dans le sang carotidien à une valeur de $28,1 \pm 4,7 \mathrm{pM}$, au temps $15 \mathrm{~min}$, avec un taux basal de $8,1 \pm 1,2 \mathrm{pM}$, et atteignant au temps 30 min une valeur maximale de $36,1 \pm 8,5 \mathrm{pM}$. Un plateau de sécrétion de l'ordre de $30 \mathrm{pM}$ était ensuite enregistré jusqu'à la fin de la période de stimulation. L'interruption de la perfusion de glucose s'est traduite par un retour rapide du taux de CCK circulante à une valeur proche de celle observée en période basale (figure 3 ).

\section{DISCUSSION}

Dans le travail présent, en périodes basale et de stimulation, les taux de CCK portale
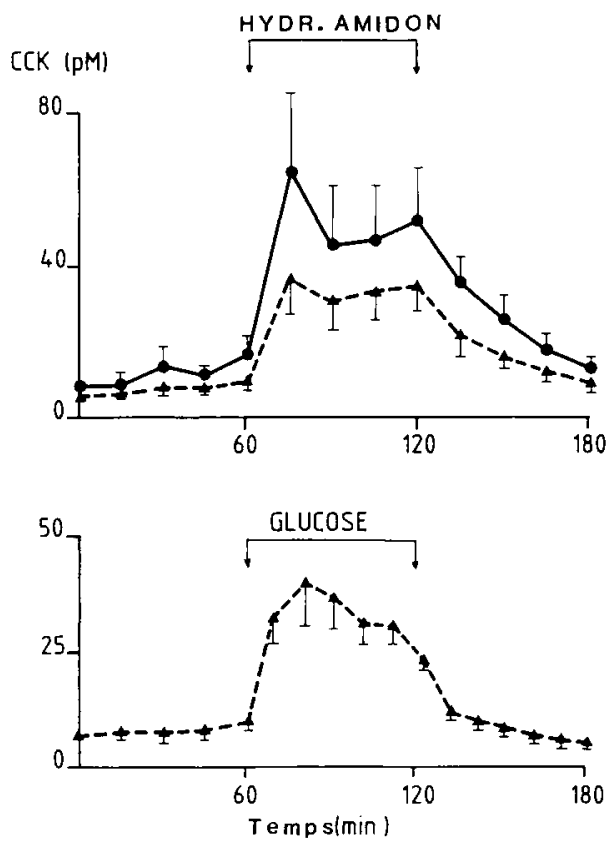

Fig 3. Effet de l'administration duodénale d'un hydrolysat d'amidon (tracé du haut) ou de glucose (tracé du bas) sur le taux plasmatique de CCK dans la veine porte et dans l'artère carotide (moyenne en $\mathrm{pM} \pm$ écart type réduit, $\mathrm{n}=6$ ). Le taux plasmatique de CCK dans la veine porte n'a pas été déterminé lors des manipulations avec le glucose.

étaient systématiquement supérieurs d'environ $30 \%$ aux valeurs correspondantes de la CCK périphérique mettant ainsi en évidence une libération de peptide depuis l'aire splanchnique. Cependant, le gradient porto-artériel est modeste, ce qui suggère que le foie ne joue pas un rôle majeur dans la dégradation de la CCK immunoréactive. Ce résultat est en accord avec un travail récent réalisé chez le porc anesthésié qui montrait que le rein était le site préférentiel de dégradation de la CCK plasmatique alors que le rôle du foie était moindre (Cuber et al, 1989a). 
Les triglycérides contenant des acides gras à chaîne longue sont de puissants stimulants de la sécrétion de CCK chez l'homme (Hopman et al, 1985; Liddle et al, 1985; Miazza et al, 1985) et chez le chien (Fried et al, 1983; Shiratori et al, 1986) alors que seul un acide gras à chaîne courte (acide octanoïque) est capable d'induire une sécrétion transitoire de CCK chez le rat (Cuber et al, 1989e). Le travail présent montre que l'augmentation du taux de CCK est tardive, la valeur maximale étant atteinte à la fin de la perfusion. Cette latence pourrait correspondre au temps nécessaire à l'hydrolyse des triglycérides par la lipase pancréatique, les acides gras libérés exerçant alors leur effet sécrétagogue. Un travail récent réalisé chez le chien soumis à une ligature du canal pancréatique (Watanabe et al, 1984) montre que, dans ces conditions, les triglycérides ne stimulent pas la libération de CCK, alors que l'acide oléique a un effet très marqué. Au total, il semble donc bien que l'élévation du taux plasmatique de CCK résultant de la perfusion de triglycérides est la conséquence de l'apparition des produits d'hydrolyse dont la nature reste à préciser. $\mathrm{Ce}$ même mécanisme semble également s'appliquer chez le porc à 2 autres hormones intestinales, la neurotensine (Cuber et al, 1989c) et le gastric inhibitory polypeptide (Cuber et al, communication personnelle).

Les protéines entières, les hydrolysats protéiques et les acides aminés sont de puissants stimulants de la sécrétion de CCK chez l'homme (Liddle et al, 1985; Miazza et al, 1985). Parmi les acides aminés, le tryptophane et la phénylalanine sont les meilleurs sécrétagogues au moins chez le chien (Chang et Chey, 1983). Chez le rat, les protéines (Liddle et al, 1986) et les hydrolysats protéiques (Cuber et al, 1989e) induisent une sécrétion soutenue de CCK alors que les acides aminés sont sans effet. Les mécanismes qui sous- tendent les réponses sécrétoires aux protéines et aux hydrolysats protéiques semblent être différents. Les protéines comme la caséine qui offrent la particularité d'être de bons substrats de la trypsine, stimuleraient la libération de CCK en levant l'inhibition de la sécrétion de peptide imposée par la trypsine lorsque celle-ci est présente seule (c'est-à-dire en l'absence du substrat) à l'état basal. Cette théorie, proposée par Green et al (1973), n'est pas complètement admise. II a été démontré par exemple que certaines protéines comme l'albumine bovine sont de bons substrats de la trypsine, alors que l'administration orale de cette protéine ne conduit à aucune variation significative des taux plasmatiques de CCK. Par ailleurs, à l'aide d'un modèle de duodéno-jéjunum isolé vascularisé de rat, la perfusion luminale d'une solution mixte de nutriments ou d'hydrolysats protéiques divers dans un segment ne contenant plus de trypsine déclenche une sécrétion soutenue de CCK dans le sang portal (Cuber et al, 1989d, 1989e).

II apparaît donc que la trypsine luminale mais également les nutriments par euxmêmes sont capables de moduler la sécrétion de CCK chez le rat. Une situation analogue semble également prévaloir chez l'homme (Owyang et al, 1986). Chez le porc, la trypsine luminale ne semble pas jouer un rôle majeur dans le contrôle de la sécrétion de CCK. La dérivation du suc pancréatique chez l'animal muni d'une fistule pancréatique chronique n'est, en effet, accompagnée que d'une augmentation modeste et transitoire du taux de CCK portale, variation non reflétée dans le sang périphérique (Corring et al, 1985). Au total, la sécrétion de CCK induite par les aliments résulterait d'une interaction directe des nutriments avec la cellule endocrine dans cette espèce. Le travail présent montre qu'un hydrolysat protéique ne pro- 
voque qu'une libération brève de CCK malgré la persistance de la perfusion du nutriment. Ce résultat exclut donc un effet majeur des acides aminés une fois libérés des chaînes polypeptidiques sur la sécrétion de CCK. Le caractère transitoire de la libération de CCK consécutive à l'instillation duodénale d'hydrolysat protéique n'a pas trouvé d'explication satisfaisante. Cependant, l'observation que la perfusion de ce nutriment provoque une sécrétion soutenue de somatostatine (Cuber et al, communication personnelle) suggère que la sécrétion de CCK pourrait être inhibée par la somatostatine, soit par une voie hormonale, soit par un effet paracrine. La démonstration d'un puissant effet inhibiteur de la somatostatine sur la libération de CCK a été faite chez l'homme et chez le chien (Schlegel et al, 1977; Konturek et al, 1987).

Les glucides ont, tout au plus, un effet très modeste sur la sécrétion de CCK chez le rat (Liddle et al, 1986; Douglas et al, 1988) alors que les résultats obtenus chez I'homme sont divergents. L'administration orale de glucose provoque une élévation nette de la concentration plasmatique de CCK (Liddle et al, 1985) alors que la perfusion luminale d'un hydrolysat d'amidon contenant des chaînes glucidiques à faible degré de polymérisation est sans effet sur la CCK (Miazza et al, 1985). L'étude présente menée avec cette même source glucidique montre une élévation rapide et de grande amplitude des taux de CCK portale et périphérique qui restent à des valeurs élevées jusqu'à la fin de la perfusion. Un profil de libération de CCK similaire est observé lorsque le glucose est administré par voie duodénale. On peut en conclure que le glucose, produit terminal de l'hydrolyse de l'amidon, est un puissant stimulant de la sécrétion de CCK mais un effet direct des chaînes glucidiques sur les cellules I ne peut être exclu.
Au plan de la physiologie digestive du porc, ce résultat est particulièrement intéressant eu égard aux habitudes alimentaires de cet animal. La ration journalière d'un porc en croissance est constituée à $80 \%$ de glucides totalement digestibles (amidon), à $14 \%$ de protéines et à $2 \%$ de lipides. Compte tenu de l'effet modeste des protéines sur la sécrétion de CCK et de l'apport faible en lipides de la ration qui ne peuvent donc pas avoir une incidence majeure sur la sécrétion de CCK, les glucides de la ration sont largement responsables de la libération de CCK observée après l'ingestion d'un repas standard (Cuber et al, 1989b). L'incrément de CCK dans le sang périphérique lors de la perfusion de lipides ou de glucides est de l'ordre de 30 pM alors que l'ingestion d'un repas standard provoque une augmentation de la concentration de CCK plasmatique d'environ $10 \mathrm{pM}$ (Cuber et al, 1989b). Le taux plasmatique seuil de $\mathrm{CCK}$ induisant un effet stimulant significatif sur le pancréas exocrine est de $100 \mathrm{pM}$ (Cuber et al, 1989b). On peut en conclure que, chez le porc, la variation du taux de CCK induite soit par un repas standard, soit par une perfusion luminale de nutriments, n'est pas suffisante pour stimuler le pancréas exocrine à moins que l'effet de la CCK soit potentialisé par d'autres hormones comme la neurotensine qui stimule la sécrétion du pancréas exocrine (Cuber et al, 1989c).

\section{RÉFÉRENCES}

Cantor P, Rehfeld JF (1989) Cholecystokinin in pig plasma: release of components devoid of a bioactive $\mathrm{COOH}$-terminus. Am $J$ Physiol 256, G53-G61

Chang TM, Chey WY (1983) Radioimmunoassay of cholecystokinin. Dig Dis Sci $28,456-$ 468

Corring T, Chayvialle JA, Simoes-Nunes C, Abello $J$ (1985) Régulation de la sécrétion 
pancréatique par rétroaction négative et hormones gastro-intestinales plasmatiques chez le porc. Reprod Nutr Dév 25, 439-450

Cuber JC, Bernard C, Gibard T, Chayvialle JA (1989a) Pharmacokinetics and organ catabolism of cholecystokinin octapeptide in pigs. Regul Pept 26, 203-213

Cuber JC, Corring T, Levenez F, Bernard C, Chayvialle JA (1989b) Effects of cholecystokinin octapeptide on the pancreatic exocrine secretion in the pig. Can J Physiol Pharmacol 67, 1391-1397

Cuber JC, Philippe C, Abello J, Corring T, Levenez F, Chayvialle JA (1989c) Plasma neurotensin in the conscious pig: release by individual food components and effects on the exocrine pancreas secretion. Pancreas (sous presse)

Cuber JC, Vilas F, Charles N, Bernard C, Chayvialle JA (1989d) Bombesin and nutrients stimulate release of CCK through distinct pathways in the rat. Am J Physiol 256, G989G996

Cuber JC, Bernard G, Fushiki T, Bernard C, Yamanishi $R$, Sugimoto $E$, Chayvialle JA (1989e) Luminal CCK-releasing factors in the isolated vascularly perfused rat duodenojejunum. Am J Physiol (sous-presse)

Douglas BR, Woutersen RA, Jansen JBMJ, de Jong AJL, Lamers CBHW (1988) The influence of different nutrients on plasma cholecystokinin levels in the rat. Experientia (Basel) 44, 21-23

Eberlein GA, Eysselein VE, Lee TD, Shively JE, Davis $M$, Schaeffer $M$, Niebel $W$, Zeeh J, Moessner A, Meyer HE, Grandt D, Goebell $H$, Reeve JR (1989) Processing of human preprocholecystokinin by signal peptidase: formation of cholecystokinin-83. Gastroenterology $96, \mathrm{~A} 134$

Eng J, Du BH, Pan YCE, Chang M, Hulmes JD, Yalow RS (1984) Purification and sequencing of a rat intestinal 22 amino acid Cterminal CCK fragment. Peptides 5, 12031206

Eysselein VE, Böttcher W, Kauffman GL, Walsh $\mathrm{JH}$ (1984) Molecular heterogeneity of canine cholecystokinin in portal and peripheral plasma. Regul Pept 9, 173-185

Fölsch UR, Cantor P, Wilms HM, Schafmayer A, Becker HD, Creutzfeldt W (1987) Role of cholecystokinin in the negative feedback control of pancreatic enzyme secretion in conscious rats. Gastroenterology 92, 449458

Fourmy D, Pradayrol L, Antoniotti $H$, Esteve JP, Ribet A (1982) Purification of radio-iodinated cholecystokinin peptides by reverse phase HPLC. J Liq Chromatogr 5, 755-766

Fried GM, Ogden WD, Swierczek J, Greeley $\mathrm{GH}$, Rayford PL, Thompson JC (1983) Release of cholecystokinin in conscious dogs: correlation with simultaneous measurements of gallbladder pressure and pancreatic protein secretion. Gastroenterology 85, 11131119

Green GM, Olds BA, Mathews G, Lyman RL (1973) Protein as a regulator of pancreatic enzyme secretion in the rat. Proc Soc Exp Biol Med 142, 1162-1167

Hopman WPM, Jansen JBMJ, Lamers CBHW (1985) Comparative study of the effects of equal amounts of fat, protein, and starch on plasma cholecystokinin in man. Scand J Gastroenterol 20, 843-847

Konturek SJ, Konturek JW, Lamers CB, Tasler $J$, Bilski J (1987) Role of secretion and CCK in the stimulation of pancreatic secretion in conscious dogs: effects of atropine and somatostatin. Int J Pancreatol 2, 223-235

Liddle R, Goldfine I, Williams J (1984) Bioassay of plasma cholecystokinin in rats: effects of food, trypsin inhibitor, and alcohol. Gastroenterology 87, 542-549

Liddle RA, Goldfine ID, Rosen MS, Taplitz RA, Williams JA (1985) Cholecystokinin bioactivity in human plasma. $J$ Clin Invest 75,1144 1152

Liddle RA, Green GM, Conrad CK, Williams JA (1986) Proteins but no amino acids, carbohydrates, or fats stimulate cholecystokinin secretion in the rat. Am J Physiol 251, G243G248

Louie DS, May D, Miller P, Owyang C (1986) Cholecystokinin mediates feedback regulation of pancreatic enzyme secretion in rats. Am J Physiol 250, G252-G259

Miazza B, Palma R, Lachange JR, Chayvialle JA, Jonard PP, Modigliani R (1985) Jejunal secretory effect of intraduodenal food in humans. Gastroenterology 88, 1215-1222

Mutt V, Jorpes JE (1968) Structure of porcine cholecystokinin pancreozymin 1-cleavage with thrombin and with trypsin. Eur J Biochem 6, 156-162 
Ondetti MA, Rubin B, Engel SL, Pluscec J, Scheehan JT (1970) Cholecystokininpancreozymin: recent developments. $A m J$ Dig Dis 15, 149-156

Owyang C, Louie DS, Tatum D (1986) Feedback regulation of pancreatic enzyme secretion. J Clin Invest 77, 2042-2047

Reeve JR, Eysselein VE, Walsh JH, Sankaran $H$, Deveney CW, Tourtellotte WW, Miller C, Shively JE (1984) Isolation and characterization of biologically active and inactive cholecystokinin-octapeptides from human brain. Peptides 5, 959-966

Rérat A, Simoes Nunes C, Mendy F, Roger $L$ (1988) Amino acid absorption and production of pancreatic hormones in non-anesthetized pigs after duodenal infusions of a milk enzymic hydrolysate of free amino acids. $B r J$ Nutr 60, 121-136

Schlegel W, Raptis S, Harvey F, Oliver JM, Pfeiffer F (1977) Inhibition of cholecystokinin- pancreozymin release by somatostatin. Lancet 2 (8030), 166-168

Shiratori $K$, Watanabe $S$, Chey WY, Lee KY, Chang TM (1986) Endogenous cholecystokinin drives gallbladder emptying in dogs. $A m J$ Physiol 251, G553-G558

Tatemoto $K$, Jörnvall $H$, Siimesmaa $S$, Hallden G, Mutt $\vee(1984)$ Isolation and characterization of cholecystokinin-58 (CCK-58) from porcine brain. Febs Lett 174, 289-293

Walsh JH (1987) Gastrointestinal hormones. In: Physiology of the gastrointestinal tract (Johnson LR, ed), Raven Press, New York, 181253

Watanabe S, Chey WY, Lee KY, Chang TM (1984) Importance of pancreatic enzyme on release of endogenous CCK and exocrine pancreatic secretion in response to fat in dog. Gastroenterology 86, 1293 